\title{
EQUILIBRIA AND STABILITY IN SET-VALUED ANALYSIS: A VIABILITY APPROACH
}

\author{
PATRICK SAINT-PIERRE ${ }^{1)}$ \\ CEREMADE, CNRS U.R.A. 749 \\ Université Paris IX - Dauphine, France
}

1. Introduction. Let be $X=\mathbb{R}^{n}$. We consider the general control problem with a priori feebacks:

$$
\begin{cases}x^{\prime}(t)=f(x(t), u(t)), & \text { for almost all } t \geq 0 \\ u(t) \in U(x(t)), & \text { for all } t \geq 0\end{cases}
$$

$K \subset X$ describes the set of state constraints.

Let be $F$ the set-valued map defined by $F(x):=\{f(x, u), u \in U(x)\}$ and denote $S_{F}(x)$ the set of all solutions to the dynamical system (1) starting from a point $x$. A solution $x(\cdot) \in S_{F}(x)$ is viable if and only if

$$
\forall t \geq 0, x(t) \in K \text {. }
$$

Our aim is to study several features of control problems, more generally of systems described through differential inclusion, with state constraints:

1. We give stability properties of approximations of the Viability Kernel with discrete or fully discrete viability kernel:

$$
\operatorname{Viab}_{F}(K):=\left\{x \in K \mid \exists x(\cdot) \in S_{F}(x), x(t) \in K, \forall t\right\} .
$$

2. We apply this result to compute the minimal time control problem with state constraints and we prove the convergence of a sequence of functions to the minimal time function.

3. We consider the general problem of finding equilibria of upper semi continuous setvalued maps $F: X \rightsquigarrow Y$ :

$$
\operatorname{Equilibria}(F):=\{x \in X \mid 0 \in F(x)\}
$$

1991 Mathematics Subject Classification: Primary 46C20; Secondary 32G81.

1) Acknowledgements: it is our pleasure to thank Sławomir Plaskacz for his pertinent advice and valuable remarks.

The paper is in final form and no version of it will be published elsewhere. 
We characterize this set through viability theory, proving that it is related to the viability kernel for a particular dynamic defined in the product space $X \times Y$.

4. We extend this approach by introducing the principle of exploration methods. This has many applications. Mainly we study an algorithm of approximation of a Lyapunov function allowing asymptotic stabilization of dynamical system and an algorithm of approximation the asymptotic attraction basin of Equilibria.

2. Viability Kernel. Let us define:

$$
F(x):=\{f(x, u) \mid u \in U(x)\} .
$$

A closed subset $D$ is a Viability Domain for $F$ if and only if $D$ enjoys the viability property:

$$
\forall x \in D, \exists x(\cdot) \in S_{F}(x) \mid x(t) \in D, \quad \forall t \geq 0
$$

and the Viability Kernel of $K$ for $F$ is the largest closed viability domain for $F$ contained in $K$. Let $T_{D}(x)$ be the Bouligand contingent cone ${ }^{2}$ at $x$ to $D$. We recall that

THEOREM 2.1. Let $F$ be a Marchaud ${ }^{3}$ map and $D$ be a closed subset of $X$. The following properties are equivalent:

$$
\begin{aligned}
& D \text { is a viability domain, } \\
& \forall x \in D, F(x) \cap T_{D}(x) \neq \emptyset, \\
& \forall x \in D, \exists w \in F(x) \mid \forall p \in N P_{D}(x),\langle p, w\rangle \leq 0,
\end{aligned}
$$

where $N P_{D}(x)$ denotes the set of proximal normals ${ }^{4}$ to $D$ at $x$.

This geometric condition (3) can be exploited thanks to a discretization process. Indeed, with the system $x^{\prime} \in F(x)$ we associate a suitable approximation $F_{\varepsilon}$ of $F$ and the discrete system:

which can be equivalently written:

$$
\frac{x^{n+1}-x^{n}}{\varepsilon} \in F_{\varepsilon}(x)
$$

$$
x^{n+1} \in G_{\varepsilon}\left(x^{n}\right):=x^{n}+\varepsilon F_{\varepsilon}\left(x^{n}\right) .
$$

A closed subset $D$ is a Discrete Viability Domain for $G_{\varepsilon}$ if and only if $D$ enjoys the discrete viability property:

$$
\forall x \in D, \exists\left(x^{n}\right)_{n \in \mathbb{N}} \text { solution to (5) such that } x^{n} \in D, \forall n \in \mathbb{N} \text {. }
$$

We define the Discrete Viability Kernel of $K$ for $G_{\varepsilon}$ which is the largest closed discrete viability domain for $G_{\varepsilon}$ contained in $K$ satisfying:

$$
\overrightarrow{\operatorname{Viab}}_{G_{\varepsilon}}(K)=\left\{x^{0} \in K \mid \exists \text { solution }\left(x^{n}\right)_{n}, x_{n} \in K, \forall n \in \mathbb{N}\right\} .
$$

We can already prove the following:

\footnotetext{
${ }^{2} T_{D}(x):=\left\{v \in X \mid \lim _{h \rightarrow 0}(1 / h) d_{D}(x+h v)=0\right\}$.

$3 F$ is Marchaud if and only if $F$ is upper semicontinuous with compact convex nonempty values and linear growth.

${ }^{4} N P_{D}(x):=\left\{p \in X \mid x \in \Pi_{D}(x+p)\right\}$, where $\Pi_{D}$ denote the euclidian projection onto $D$.
} 
THEOREM 2.2. Let $F$ be a Marchaud set-valued map and $K$ a closed set. Let be $M(x):=\max _{y \in B(x, 1) \cap K}\|F(y)\|$ and consider any approximation $F_{\varepsilon}(x)$ of $F$ satisfying:

$$
\begin{aligned}
& \operatorname{Limsup} \operatorname{Graph}\left(F_{\varepsilon}\right) \subset \operatorname{Graph}(F) \\
&\left.\forall \epsilon \in] 0, \frac{1}{M(x)}\right], \quad F\left(x+\varepsilon M(x) \mathcal{B}_{X}\right) \subset F_{\varepsilon}(x)
\end{aligned}
$$

Let be $G_{\varepsilon}(x):=x+\varepsilon F_{\varepsilon}(x)$. Then

$$
\lim _{\varepsilon \rightarrow 0}{\overrightarrow{\operatorname{Viab}_{G}}}_{G_{\varepsilon}}(K)=\operatorname{Viab}_{F}(K)
$$

Proof. The proof is a consequence of Proposition 2.3 and Proposition 2.5.

Proposition 2.3. Let $F$ be a Marchaud map and $K$ be a closed subset. Let $F_{\varepsilon}$ be an approximation of $F$ and $K_{\varepsilon}$ an approximation of $K$ satisfying assumption (6). Let $V_{\varepsilon}$ be a discrete viability domain for $G_{\varepsilon}$.

Then $V^{\sharp}:=\operatorname{Limsup}_{\varepsilon \rightarrow 0} V_{\varepsilon}$ is a viability domain for $F$.

Proof. Let us consider $x \in V^{\sharp}$ and $p \in N P_{V^{\sharp}}(x)$. Let $\varepsilon_{n} \rightarrow 0$ be a sequence such that $x_{n} \in V_{\varepsilon_{n}}$ exists converging to $x$ and consider ${ }^{5} z_{n} \in \Pi_{V_{\varepsilon_{n}}}(x+p)$.

LEMMA 2.4. We have: $\lim _{n \rightarrow \infty} z_{n}=x$.

Proof.6 We have

$$
\left\|x-z_{n}\right\|^{2}=\left\|x-x_{n}\right\|^{2}+2\left\langle z_{n}-x_{n}, p\right\rangle+2\left\langle x_{n}-z_{n}, x+p-z_{n}\right\rangle-\left\|x_{n}-z_{n}\right\|^{2}
$$

and since $x_{n} \in V_{\varepsilon_{n}}$, we have also

$$
\left\langle x_{n}-z_{n}, x+p-z_{n}\right\rangle \leq 0
$$

then

$$
\left\|x-z_{n}\right\|^{2} \leq\left\|x-x_{n}\right\|^{2}+2\left\langle z_{n}-x_{n}, p\right\rangle .
$$

Let be $z$ any element belonging to $\operatorname{Adh}\left(z_{n}\right)$, the set of adherence values of the sequence $\left(z_{n}\right)$. From the very definition of $V^{\sharp}$, since $x \in \Pi_{V^{\sharp}}(x+p)$ we have:

$$
\langle z-x, p\rangle \leq 0 \text {. }
$$

Then when $n \rightarrow \infty$ we deduce from (9) that $\|x-z\|^{2} \leq 0$.

On one hand, since $V_{\varepsilon_{n}}$ is a discrete viability domain, there exists $v_{n} \in G_{\varepsilon_{n}}\left(z_{n}\right) \cap V_{\varepsilon_{n}}$ and since $z_{n}$ is the projection of $x+p$ on $V_{\varepsilon_{n}}$, we have

$$
\left\|z_{n}-(x+p)\right\| \leq\left\|v_{n}-(x+p)\right\| .
$$

On the other hand, from the definition of $G_{\varepsilon}$ there exists $w_{n} \in F_{\varepsilon_{n}}\left(z_{n}\right)$ such that $v_{n}=$ $z_{n}+\varepsilon_{n} w_{n}$. Then we obtain:

$$
\begin{aligned}
\left\|z_{n}-(x+p)\right\|^{2} & \leq\left\|z_{n}-(x+p)+\varepsilon_{n} w_{n}\right\|^{2} \\
& =\left\|z_{n}-(x+p)\right\|^{2}+2\left\langle z_{n}-(x+p), \varepsilon_{n} w_{n}\right\rangle+\varepsilon_{n}^{2}\left\|w_{n}\right\|^{2}
\end{aligned}
$$

\footnotetext{
${ }^{5}$ We can assume that $z_{\varepsilon_{n}}$ is unique since, if not, one can replace $p$ by $p^{\prime}=1 / 2 p$ and then the projection becomes unique.

6 This version of the proof is a revisited version by P. Cardaliaguet.
} 
So

Let $n$ tend to $\infty$.

$$
\left\langle p, w_{n}\right\rangle \leq \frac{1}{2} \varepsilon_{n}\left\|w_{n}\right\|^{2}-\left\langle z_{n}-x, w_{n}\right\rangle
$$

Since

$$
\operatorname{Limsup}_{n \rightarrow \infty} \operatorname{Graph}\left(F_{\varepsilon_{n}}\right) \subset \operatorname{Graph}(F),
$$

from Lemma 2.4 we deduce that $\lim _{n \rightarrow \infty}\left(z_{n}, w_{n}\right)=(x, w) \in \operatorname{Graph}(F)$ exists such that $\langle p, w\rangle \leq 0$. So condition (4) of Theorem 2.2 holds true. This prove that $V^{\sharp}$ is a viability domain.

The Marchaud property of $F$ is not sufficient to prove that the viability kernel of $K$ for $F$ can be approached exactly by a sequence of discrete viability kernels. We need a stronger regularity assumption on $F$ which is satisfied when $F$ is Lipschitz but which is quite general including many situations where $F$ is only upper semicontinuous.

Proposition 2.5. Let $F$ be a Marchaud set-valued map and $D$ a viability domain for $F$. Let us consider any approximation $F_{\varepsilon}(x)$ of $F$ satisfying assumption $(7)$ and set $G_{\varepsilon}(x):=x+\varepsilon F_{\varepsilon}(x)$. Then $D$ is a discrete viability domain for $G_{\varepsilon}$

Pro of. Let be $x \in D$ and consider any solution $x(\cdot) \in S_{F}(x)$ viable in $D$. We know that

$$
\forall t \geq 0, \quad x(t)=x+\int_{0}^{t} x^{\prime}(\tau) d \tau .
$$

Let be $\theta:=\max \{t \mid x(t) \in \mathcal{B}(x, 1)\}$. Since $x^{\prime}(\tau) \in F(x(\tau))$ for almost all $\tau \in[0, \theta]$, we have:

$$
\|x(\tau)-x\| \leq \tau M(x)
$$

and so, for all $\tau \leq(1 / M(x)), x(\tau) \in \mathcal{B}(x, 1)$. In particular $x(1 / M(x)) \in \mathcal{B}(x, 1)$. So $\theta \geq(1 / M(x))$ and

$$
\forall \tau \leq \frac{1}{M(x)},\|x(\tau)-x\| \leq \tau M(x)
$$

On the other hand,

$$
x(t)-x=\int_{0}^{t} x^{\prime}(\tau) d \tau \in \int_{0}^{t} F(x(\tau)) d \tau \subset \int_{0}^{t} F(x+\|x(\tau)-x\| \mathcal{B}) d \tau
$$

and from (10) we have for all $t \leq(1 / M(x))$

$$
x(t)-x \in \int_{0}^{t} F(x+\tau M(x) \mathcal{B}) d \tau \subset t F(x+t M(x) \mathcal{B}) .
$$

Then from assumption (7), for all $x \in D$ and for all $\varepsilon \leq(1 / M(x))$, we have

$$
x(\varepsilon) \in x+\varepsilon F(x+\varepsilon M(x) \mathcal{B}) \subset x+\varepsilon F_{\varepsilon}(x)=G_{\varepsilon}(x) .
$$

So, since $x_{\varepsilon} \in D$, we have proved that

$$
G_{\varepsilon}(x) \cap D \neq \emptyset
$$

that is to say that $D$ is a discrete viability domain for $G_{\varepsilon}$. 
Corollary 2.6. Let be $F$ a Marchaud and $\ell$-Lipschitz map. Assume that $M:=$ $\sup _{x \in K} M(x)$ is finite. Let be $F_{\varepsilon}(x):=F(x)+(M \ell / 2) \varepsilon \mathcal{B}$ and $G_{\varepsilon}:=x+\varepsilon F_{\varepsilon}(x)$. Then

$$
\operatorname{Viab}_{F}(K)=\lim _{\varepsilon \rightarrow 0}{\overrightarrow{\operatorname{Viab}_{G \varepsilon}}}_{G_{\varepsilon}}(K)
$$

Proof. Assumption (6) is satisfied for $F_{\varepsilon}$. On the other hand, for any $x \in \operatorname{Viab}_{F}(K)$ and for any viable solution $x(\cdot) \in \mathcal{S}_{F}(x)$, from inclusion (11) we get:

$$
x(\varepsilon) \in x+\varepsilon F(x)+\int_{0}^{\varepsilon} M \ell \tau \mathcal{B} d \tau=x+\varepsilon F(x)+\frac{M \ell}{2} \varepsilon^{2} \mathcal{B}:=x+\varepsilon F_{\varepsilon}(x)
$$

and so

$$
x(\varepsilon) \in G_{\varepsilon}(x) \cap \operatorname{Viab}_{F}(K) \neq \emptyset .
$$

The viability kernel of $K$ for $F$ is a discrete viability domain for $G_{\varepsilon}$ contained in $\overrightarrow{\mathrm{Viab}}_{G_{\varepsilon}}(K)$. From Proposition 2.3, equality ensues.

The Viability Kernel Algorithm. To compute the discrete viability kernel of $K$ for $G$ we need the following

Proposition 2.7. Let $G: X \rightsquigarrow X$ be an upper semicontinuous set-valued map with compact values and $K$ be a closed set. Consider the decreasing sequence of sets $K^{n}$ defined by

$$
\left\{\begin{array}{l}
K^{0}:=K \\
K^{n+1}:=\left\{x \in K^{n} \mid G(x) \cap K^{n} \neq \emptyset\right\} .
\end{array}\right.
$$

Let be $K^{\infty}:=\lim _{n \rightarrow \infty} K^{n}=\bigcap_{n=0}^{\infty} K^{n}$. Then

$$
K^{\infty}={\overrightarrow{\operatorname{Viab}_{G}}}_{(K)}
$$

One can find in [10] the fully discrete algorithm and convergence results which allow to compute fully discrete viability kernels defined on grids ${ }^{7}$.

\section{Applications}

3.1. Calculus of the minimal time function for constrained control problems. Amongst many applications we show how this result can be applied to prove the convergence of algorithms which allow approximation of the minimal time function for target problems with state constraints and without local controllability assumptions on a neighbourhood of the target. This is a joint work with P. Cardaliaguet and M. Quincampoix (see [5] for control problem and [7] for Differential Games)

${ }^{7}$ We call grid of size $h$ any countable subset $X_{h}$ of points of $X$ satisfying the following property: $\forall x \in X \exists x_{h} \in X_{h}$ such that $d\left(x, x_{h}\right) \leq h$ 
Let be $C$ a closed target and $K$ the set of constraints and consider the system:

$$
\begin{array}{rlrl}
\left(x^{\prime}, y^{\prime}\right) & = \begin{cases}(f(x, u),-1) & \text { if } x \notin C, \\
\overline{\operatorname{Co}}\{(f(x, u),-1),(0,0)\} & \text { if } x \in C,\end{cases} \\
u & \in U(x), & \\
(x(t), y(t)) & \in K \times \mathbb{R}^{+} & \forall t \geq 0 .
\end{array}
$$

Let us denote $F$ the set-valued map $x \rightsquigarrow F(x):=\{f(x, u), u \in U(x)\}$ The minimal time — or hitting time — function is defined by:

$$
\vartheta_{C}^{K}\left(x_{0}\right):=\inf _{x(\cdot) \in S_{F}\left(x_{0}\right)}\{\tau \mid x(\tau) \in C, x(t) \in K, \forall t \leq \tau\}
$$

Proposition 3.1. If $F$ is Marchaud then:

- $\vartheta_{C}^{K}(\cdot)$ is lower semicontinuous,

- the infimum value is reached for some solution.

We refer to [5] for the proof of this Proposition.

3.1.1. Characterization through Viability Theory. Let us denote $Z:=X \times \mathbb{R}$ and $\Phi$ the expanded set-valued map defined by:

$$
\Phi(x, y)= \begin{cases}F(x) \times\{-1\} & \text { if } x \in D \\ \overline{\mathrm{Co}}((F(x) \times\{-1\}) \cup(\{0\} \times\{0\})) & \text { if } x \in C\end{cases}
$$

and consider the differential inclusion

$$
\left(x^{\prime}(t), y^{\prime}(t)\right) \in \Phi(x(t), y(t)), \quad \text { a.e. } t \geq 0 .
$$

Let us note that if $F$ is a Marchaud set-valued map, $\Phi$ is also Marchaud. We have the following:

Theorem 3.2 Let $F: X \rightsquigarrow X$ be a Marchaud map, $K$ and $C$ two closed subsets of $X$. We set

$$
\mathcal{H}:=\left\{(x, y) \in(K \cap \overline{X \backslash C}) \times \mathbb{R}^{+}\right\}
$$

Let $\vartheta_{C}^{K}(\cdot)$ be the hitting time function of $C$ for solutions in $\mathcal{S}_{F}(\cdot)$ which remains in $K$. Then the epigraph of $\vartheta_{C}^{K}(\cdot)$ is the viability kernel of $\mathcal{H}$ for $\Phi$ :

$$
\operatorname{Viab}_{\Phi}(\mathcal{H})=\operatorname{Epi}\left(\vartheta_{C}^{K}\right)
$$

3.1.2. Algorithm. For the sake of simplicity, we consider in this section the case when $F$ is $\ell$-Lipschitz and $M$-bounded.

Fix $\tau>0$ and let us now define the following nondecreasing sequence of maps defined on $K$ :

$$
\begin{aligned}
\vartheta_{\tau}^{0}(x) & := \begin{cases}0 & \text { if } x \in K \\
+\infty & \text { if } x \notin K\end{cases} \\
\vartheta_{\tau}^{k+1}(x) & := \begin{cases}\tau+\inf _{u \in U,\|w\| \leq 1}\left\{\vartheta_{\tau}^{k}\left(x+\tau f(x, u)+\frac{M \ell \tau^{2}}{2} w\right)\right\} & \text { if } d_{C}(x)>M \tau \\
0 & \text { if } d_{C}(x) \leq M \tau .\end{cases}
\end{aligned}
$$

From the Convergence Theorem, we deduce the following convergence result:

TheOREM 3.3. Under the asumptions of Theorem $3.2 \vartheta_{\tau}^{\infty}(x):=\lim _{k \rightarrow \infty} \vartheta_{\tau}^{k}(x)$ exists and we have

$$
\forall x \in K, \lim _{\tau \rightarrow 0} \vartheta_{\tau}^{\infty}(\cdot)=\vartheta_{C}^{K}(\cdot)
$$


Proof. We set

$$
\Phi(x, y):= \begin{cases}\{F(x)\} \times\{-1\} & \text { if } x \notin C \\ \overline{\operatorname{Co}}[\{F(x)\} \times\{-1\} \cup\{0,0\}] & \text { otherwise }\end{cases}
$$

and we introduce

$$
\Gamma_{\tau}(x, y):= \begin{cases}(x, y)+\tau\left[\left\{F(x)+\frac{M \ell \tau}{2} \mathcal{B}\right\} \times\{-1\}\right] & \text { if } d_{C}(x)>M \rho \\ (x, y)+\tau \overline{\mathrm{Co}}\left[\left\{F(x)+\frac{M \ell \tau}{2} \mathcal{B}\right\} \times\{-1\} \cup\{0,0\}\right] & \text { otherwise }\end{cases}
$$

Then combining Theorem 3.2 with Theorem 2.2 yields that

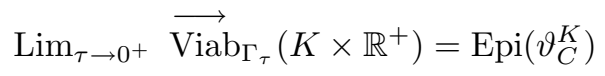

Now Proposition 2.7 states that the following decreasing sequence of closed sets:

$$
\begin{aligned}
A_{0} & :=K \times \mathbb{R}^{+} \\
A_{k+1} & :=\left\{(x, y) \in A_{k} \Gamma_{\rho}(x, y) \cap A_{k} \neq \emptyset\right\}
\end{aligned}
$$

converges to $\overrightarrow{\operatorname{Viab}}_{\Gamma_{\rho}}\left(K \times \mathbb{R}^{+}\right)$. It is easy to check that the $A_{k}$ are the epigraph of some function $\vartheta_{\tau}^{k}$. Now, exploiting the very definition of $A_{k}$ gives (15).

3.1.3. Example. In control theory, Zermelo described the problem of the swimmer that can be summed up as follows: the dynamic of the river is given by the water current function $f(x, y)$. The current is choosen to be decreasing with the distance to the middle axes of the river, with a constant direction: $f(x, y):=1-a|y|^{2}(a=0.04)$.

The swimmer has his own dynamic: he can swim in any direction at a speed $s$ whose norm ranges between $[0,0.5]$.

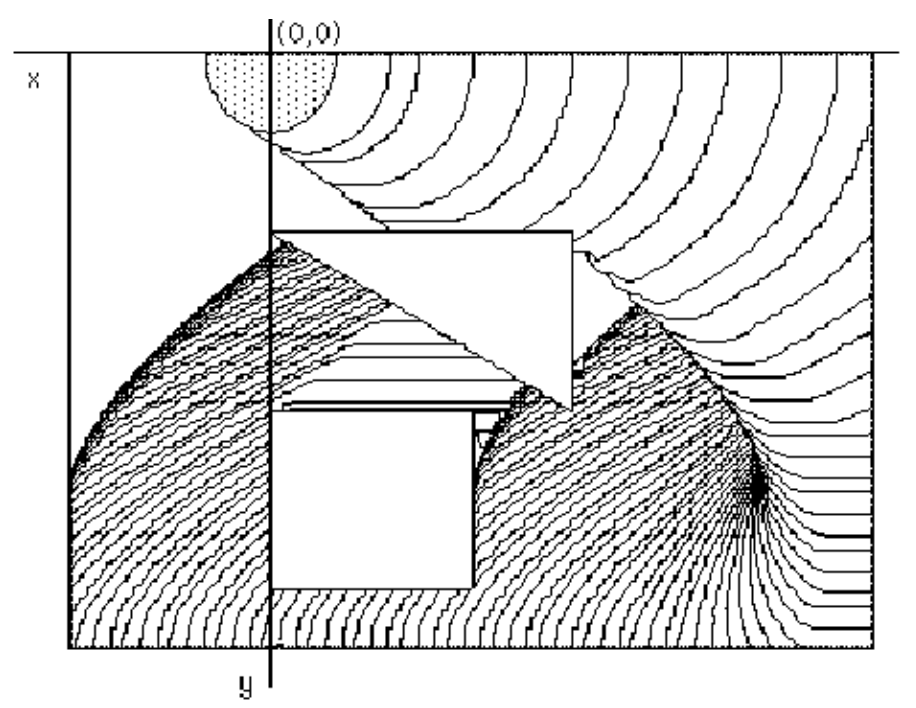

1. Minimal time function for Zermelo problem with obstacles 
Also the global dynamic is given by:

$$
\begin{aligned}
& x^{\prime}(t)=\left(1-a y^{2}\right)+u \\
& y^{\prime}(t)=v
\end{aligned}
$$$$
\text { with } u^{2}+v^{2} \leq c^{2}
$$

In the numerical example we have choosen $C=\left\{(x, y) \in \mathbb{R}^{2} \mid x^{2}+y^{2} \leq 0.44\right\}$ for the island-target and $K=[-6,2] \times[-5,5] \times \mathbb{R}^{+}$. Taking into account the symmetry of the problem, we have just viewed half part of the problem.

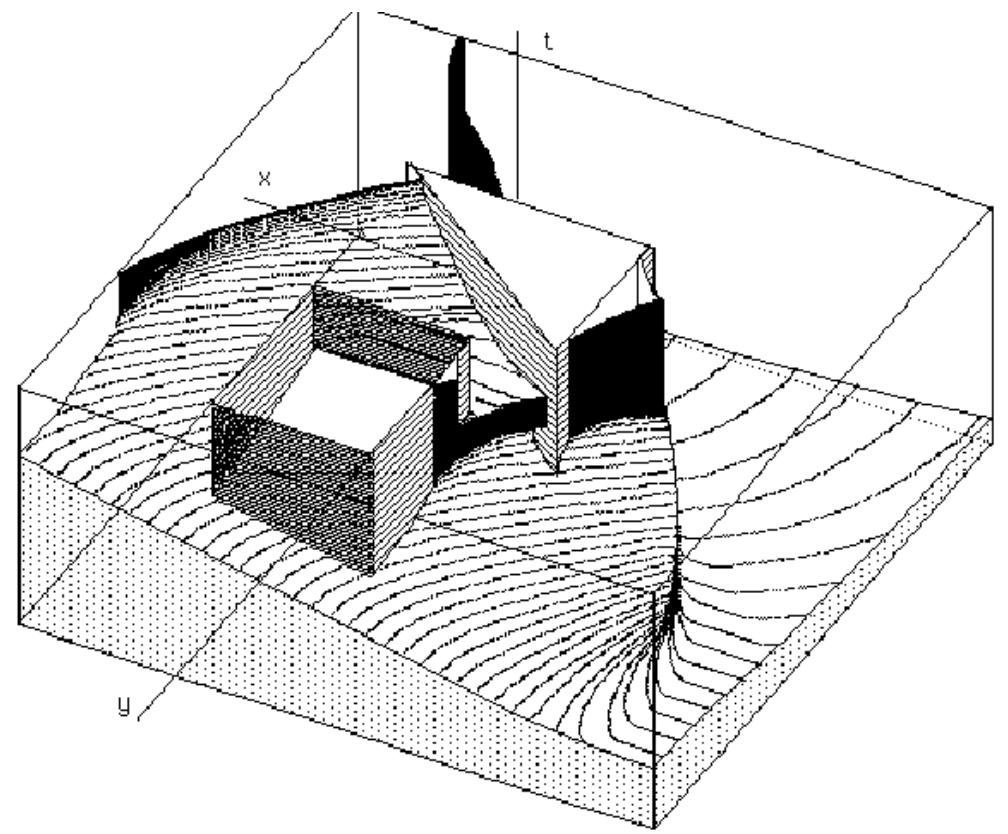

2. Level Curves of the Minimal time function for Zermelo problem with obstacles

The swimmer aims to reach an island as quickly as possible. We assume that if he pass over some "waterfall" ahead the island, he completely fails and that he has to avoid some precised area or obstacles which are shown on the figure.

The domain of the minimal time function is precisely the set of initial points $x_{0}$ from which the swimmer is able to reach the island. Out of this domain the minimal time function takes the value $+\infty$.

Let us notice that in this case the swimmer can follow up the bank since $c$ is greater than $\left(1-a|y|^{2}\right)$ whenever $y \geq y^{\star}=(1-c / a)^{1 / 2}\left(y^{\star} \simeq 3.53\right)$.

Figure 1 represents the graph and Figure 2 the level curves of the approximate minimal time function obtained for a grid of $416^{3}$ points.

A barrier phenomena can be observed showing the discontinuity of the minimal time function.

Relations with viscosity super solution of Hamilton Jacobi Bellman equations are given in [5] and [6]. 
3.2. Equilibria for set-valued dynamics. A second application of the Convergence Theorem of Viability Kernels lies in the calculus of the set of all equilibria for upper semi-continuous set-valued maps.

Let $F: X \rightsquigarrow X$ be a set-valued map and $K$ a closed subset of $X$. We denote

$$
\operatorname{Equi}_{F}(K):=\{x \in K \mid 0 \in F(x)\}
$$

the set of all equilibria for $F$ contained in $K$.

3.2.1. Characterization of the set of Equilibria. Let be $F: X \rightsquigarrow Y$ and $Z:=X \times Y$. We define $\Phi: Z \rightsquigarrow Z$ by:

$$
\Phi(x, y)=\left(\begin{array}{c}
0 \\
F(x)
\end{array}\right) .
$$

We consider the differential inclusion system

$$
\left(x^{\prime}(t), y^{\prime}(t)\right) \in \Phi(x(t), y(t)), \quad \text { a.e. } t \geq 0
$$

and for any $c \geq 0$, the constraint set:

$$
(x(t), y(t)) \in K \times c \mathcal{B}, \quad \forall t \in[0,+\infty) .
$$

Let $\Pi_{X}$ denote the projection $(x, y) \rightarrow x$.

Proposition 3.4. Let $F$ be a Marchaud set-valued map and $K \subset X$ closed. Then

$$
\forall c \in \mathbb{R}^{+} \operatorname{Equi}_{F}(K)=\Pi_{X}\left(\operatorname{Viab}_{\Phi}(K \times c \mathcal{B})\right) .
$$

For $c=0$ the result still holds true: the projection of the viability kernel of $K \times\{0\}$ is the set of equilibria of the system (18).

We can also apply Proposition 3.4 with $\phi(x):=\min _{y \in F(x)}\|y\|$, or $\phi(x):=\|f(x)\|$ when $F$ is a single-valued continuous map $f: X \rightarrow Y$, and consider the differential equation system

under the constraint

$$
\left\{\begin{array}{l}
x^{\prime}(t)=0 \\
z^{\prime}(t)=\phi(x(t))
\end{array}\right.
$$

$$
(x(t), z(t)) \in K \times[0, c], \quad \forall t \in[0,+\infty)
$$

3.3. Approximation of Lyapunov functions. In [1] J.-P. Aubin has proved the existence of a Lyapunov function greater than any given function $V$ which epigraph is the viability kernel of Epigraph $(V)$ for the corresponding dynamic $F$. We give here an algorithm which allows to compute approximation of such Lyapunov function. This method is closed to an exploration method in the epigraph of $V$.

Let $\varphi(\cdot): X \rightarrow \mathbb{R}^{+}$be a non negative function such that $\min _{x \in X} \varphi(x)=0$. The function $\varphi$ is a Lyapunov function for the differential inclusion

$$
x^{\prime}(t) \in F(x(t)), \quad \text { a.e. } t \in[0,+\infty)
$$

if there exists $x_{0} \in X$ and a solution $x^{\star}(\cdot) \in \mathcal{S}_{F}\left(x_{0}\right)$ satisfying

$$
\frac{d}{d t} \varphi\left(x^{\star}(t)\right) \leq 0, \quad \forall t \in[0,+\infty) .
$$


So if we know a Lyapunov function, we know already the behavior of the solution $x^{\star}(\cdot)$ when $t$ increases to infinity.

If equation $\varphi(x)=0$ has a unique solution $\bar{x}$, then $\lim _{t \rightarrow \infty} x^{\star}(t)=\bar{x}$.

If there exist $x_{0} \neq \bar{x}$ and $\varphi(\cdot)$ a Lyapunov function such that $x_{0} \in \operatorname{Dom}(\varphi)$, then $x_{0}$ belongs to the attraction basin of $\bar{x}$ and there exists a solution of (20) starting from $x_{0}$ which converges to $\bar{x}$. Along the solution $\varphi$ is not increasing.

The Lyapunov function $\varphi$ is not unique. For instance $\varphi(x):=0$ if $x=\bar{x}$ and $+\infty$ elsewhere is a Lyapunov function.

The following result due to J. P. Aubin states a characterization of "some best" Lyapunov function insuring asymptotic stability.

Let $V(\cdot): X \rightarrow \mathbb{R}^{+}$be a non negative function, Epi $(V)$ the epigraph of $V$ and consider the differential inclusion:

$$
\left\{\begin{array}{l}
x^{\prime}(t) \in F(x(t)) \quad \text { a.e. } t \in[0,+\infty) \\
y^{\prime}(t)=-a y(t)
\end{array}\right.
$$

Proposition 3.5. Let $F: X \rightsquigarrow X$ be a Marchaud map. Let be $K \subset X$ closed. Let $V: X \rightarrow \mathbb{R}$ such that $\operatorname{Epi}(V)$ is closed. We denote $\Phi(x, y):=(F(x),-a y)$ and $\mathcal{H}:=\operatorname{Epi}(V) \cap K \times \mathbb{R}^{+}$. Then

$$
\operatorname{Viab}_{\mathcal{H}}(\Phi)=\operatorname{Epi}(\varphi)
$$

where $\varphi(\cdot)$ is the minimal lower semi-continuous Lyapunov function greater than $V$ :

$$
\forall x \in K, \quad \varphi(x) \geq V(x) .
$$

From this Proposition, the domain of the minimal function $\varphi(\cdot)$ is the subset of initial points $x_{0}$ such that a solution exists in $\mathcal{S}_{F}\left(x_{0}\right)$, viable in $K$, which converges asymptotically to $\bar{x}$.

3.3.1. Approximation of Lyapunov Function Algorithm. The function $\varphi$ is the limit ${ }^{8}$ of a sequence of lower semicontinuous functions $\varphi^{n}$.

Let be $\varphi^{0}(\cdot)=V(\cdot)$. For all $\rho>0$ we define:

$$
\varphi^{n}(x)=\max \left[\varphi^{n}(x), \inf _{z \in F_{\rho}(x)} \frac{\varphi^{n-1}(x+\rho z)}{1-a \rho}\right] .
$$

Proposition 3.6. The sequence of functions $\left\{\varphi^{n}(\cdot)\right\}_{n}$ converges pointwisely to $\varphi(\cdot)$ as $n \rightarrow+\infty$ and $\rho \rightarrow 0$, which is the lowest lower semicontinuous Lyapunov function minorated by $V$.

Proof. From the very definition of $\varphi^{n}(\cdot)$, we have already

$$
\operatorname{Epi}\left(\varphi^{n}\right)=\left\{(x, y) \in \operatorname{Epi}\left(\varphi^{n-1}\right) \mid\left(\begin{array}{c}
x+\rho F_{\rho}(x) \\
y(1-a \rho)
\end{array}\right) \cap \operatorname{Epi}\left(\varphi^{n-1}\right) \neq \emptyset\right\} .
$$

So from the Viability Kernel Approximation Theorem we can easily check that Epi $(\varphi)$ is the viability kernel of $\operatorname{Epi}(V)$ and so that

$$
\operatorname{Viab}_{\mathcal{H}}(\Phi)=\operatorname{Epi}(\varphi)
$$

\footnotetext{
8 in the sense that its epigraph is the upper limit of the sequence of epigraphs of functions $\varphi^{n}$
} 
Moreover, since $\operatorname{Epi}\left(\varphi^{n}\right)$ is a decreasing sequence of imbedded epigraphs, for any $x \in K$ we have

$$
\varphi^{n}(x) \leq \varphi^{n+1}(x)
$$

so

$$
\varphi^{n}(x) \leq \varphi(x)
$$

On the other hand from Theorem 2.2 , since $\operatorname{Epi}(\varphi)=\operatorname{Viab}_{\mathcal{H}}(\Phi)$, we have

$$
\lim _{n \rightarrow \infty} \varphi^{n}(x) \geq \varphi(x)
$$

thus implies

$$
\lim _{n \rightarrow \infty} \varphi^{n}(x)=\varphi(x) .
$$

Let us check now that $\varphi$ is a Lyapunov function. Let be $\left(x_{0}, \varphi\left(x_{0}\right)\right) \in \operatorname{Epi}(\varphi)$. Starting from this point, there exists a solution $(x(\cdot), \varphi(x(\cdot))$ viable in $\operatorname{Epi}(\varphi)$.

Then for all $t>0,\left(x^{\prime}(t), \varphi^{\prime}(x(t)) \cdot x^{\prime}(t)\right)$ satisfies

$$
\left(x^{\prime}, \varphi^{\prime}(x) \cdot x^{\prime}\right) \in T_{\operatorname{Viab}_{\mathcal{H}}(\Phi)}(x, \varphi(x)) \cap(F(x),\{-a . \varphi(x)\}) .
$$

Then

$$
\frac{d}{d t} \varphi(x(t))=\varphi^{\prime}(x(t)) \cdot x^{\prime}(t)=-a \varphi(x(t)) \leq 0, \quad \text { p.p. } t>0
$$

so $\varphi$ is a Lyapunov function.

Lower semicontinuity derives from closedness of the viability kernel $\operatorname{Epi}(\varphi)$.

The lowest Lyapunov function property derives from the very definition of viability kernels that are maximal viability domains and from the simple remark that the epigraph of any lower semicontinuous Lyapunov function $\psi$ minorated by $V$ i a viability domain of $\operatorname{Epi}(V)$ for $\Phi$ included necessarily in $\operatorname{Epi}(\varphi)$.

3.4. Asymptotic stability for Equilibria. Let us consider the graph of a set-valued map $F$ with closed graph. A way to explore $\operatorname{Graph}(F)$ so as to find equilibria for $F$ is to consider the following dynamical system

$$
\left\{\begin{array}{l}
x^{\prime}(t) \in \mathcal{B} \\
y^{\prime}(t)=-a y(t)
\end{array}\right.
$$

and to consider the Viability Kernel of $\operatorname{Graph}(F)$ for the set-valued map $\Gamma$ defined by

$$
\Gamma(x, y):=\mathcal{B} \times\{-a y\} .
$$

It is the closed graph of a set-valued map $F^{\infty}: X \rightsquigarrow Y$ which have same equilibria than $F$.

From the very definition of the Viability Kernel, for any initial value $x_{0} \in \operatorname{Dom}\left(F^{\infty}\right)$, there exists $x(\cdot)$ such that:

$$
\forall t \geq 0, y(t)=e^{-a t} y_{0} \in F^{\infty}(x(t)) \subset F(x(t))
$$

and the limit values of $x(\cdot)$ are equilibria for $F$. 
3.4.1. Approximation of $F^{\infty}$ and convergent Algorithms. Let be $\rho>0$ and $F_{\rho}^{0}:=F$. We define $F_{\rho}^{n}$ by:

$$
F_{\rho}^{n}(x):=F_{\rho}^{n-1}(x) \bigcap\left(\frac{1}{1-a \rho} \bigcup_{u \in \mathcal{B}} F_{\rho}^{n-1}(x+\rho u)\right) .
$$

$F_{\rho}^{\infty}$ satisfies

$$
F_{\rho}^{\infty}(x) \subset \frac{1}{1-a \rho} \bigcup_{w \in B} F_{\rho}^{\infty}(x+\rho w) .
$$

Let be $y_{0} \in F_{\rho}^{\infty}\left(x^{0}\right), n$ fixed and consider the set-valued map $x \rightsquigarrow R_{\rho}^{n}(x)$ defined by

$$
R_{\rho}^{n}(x):=\left\{u \in \mathcal{B} \mid F_{\rho}^{n}(x+\rho u) \cap(1-\rho a) F_{\rho}^{n}(x) \neq \emptyset\right\} .
$$

Proposition 3.7. We have

$$
\operatorname{Graph}\left(F_{\rho}^{\infty}\right)=\operatorname{Viab}_{\Gamma}(\operatorname{Graph}(F)) .
$$

Moreover, $R_{\rho}^{n}(\cdot)$ defines a convergent algorithm: for any $x^{0} \in \operatorname{Domain}\left(R_{\rho}^{n}\right)$, the sequence defined by

$$
x^{k+1} \in x^{k}+\rho R_{\rho}^{n}\left(x^{k}\right)
$$

converges to a zero of $F_{\rho}$.

Indeed, since the viability kernel $\operatorname{Viab}_{\Phi}(K)$ associated with this problem is the set of initial points $\left(x^{0}, y^{0}\right)$ such that a solution $(x(t), y(t))$ exists satisfying $x(t) \rightarrow x^{\star}$ and $0 \in F\left(x^{\star}\right)$, the projection of $\operatorname{Viab}_{\Phi}(K)$ onto $X$ is the set of initial values $x^{0}$ for which the sequence $\left(x^{k}\right)_{k}$ defined by

$$
x^{k+1} \in x^{k}+\rho R_{\rho}^{n}\left(x^{k}\right)
$$

satifies $y^{k}=(1-\rho a)^{k} \in F_{\rho}\left(x^{k}\right)$ and so $y^{k}$ converges to zero and $x^{k}$ converges to a zero of $F_{\rho}$.

\section{References}

[1] J.-P Aubin and Byrnes, Lyapunov functions Associated with Attractors of Differential Inclusions. Cahier de Mathématiques de la Décision, (to appear).

[2] J.-P Aubin and H. Frankowska, Set-valued analysis, Birkhaüser, 1992.

[3] J.-P Aubin, Viability Theory, Birkhaüser, 1992.

[4] P. Cardaliaguet, M. Quincampoix and P. Saint-Pierre, Some Algorithms for Differential Games with two Players and one Target. To appear in Journal of Mathematical Systems, Estimation and Control, 1994.

[5] P. Cardaliaguet, M. Quincampoix and P. Saint-Pierre, Temps optimaux pour des problèmes avec contraintes et sans contrôlabilité locale, C. R. A. S. 318 (1994), 607-612.

[6] P. Cardaliaguet, M. Quincampoix and P. Saint-Pierre, Temps optimaux pour des problèmes avec contraintes et sans contrôlabilité locale (to appear).

[7] P. Cardaliaguet, M. Quincampoix and P. Saint-Pierre, Differential Games with state-constraints, Cahier de Mathématiques de la Décision, 1995.

[8] H. Frankowska, Lower semicontinuous solutions of Hamilton-Jacobi-Bellman equations, SIAM J. Control and Optimization 31 (1) (1995), 257-272. 
[9] M. Quincampoix and P. Saint-Pierre, An Algorithm for Viability Kernels in Hölderian case: Approximation by Discrete Dynamical Systems, (\& M. Quincampoix), Journal of Mathematical Systems, Estimation and Control 1993.

[10] P. Saint-Pierre, Discrete Approximation of the Viability Kernel, Applied Mathematics and Optimisation 29, 187-209. 